\title{
Nürnberger Stadtpolitik im Zeichen von Gouvernementalität und Propaganda bei Hans Rosenplüt
}

\section{Einleitung}

Der vorliegende Beitrag unternimmt den Versuch, die Darstellung des Nürnberger Stadtrats in Fastnachtspielen Hans Rosenplüts zu analysieren und damit eine für die Reichsstadt im 15. Jahrhundert charakteristische Relation zwischen Literatur und Politik nachzuzeichnen. In methodischer Hinsicht geht es dabei weniger um eine allgemeine, $d$. $h$. auf einer mittleren Ebene angesiedelte, Wechselwirkung literarischer Texte mit kulturellen Kontexten ${ }^{1}$ als vielmehr um die konkrete funktionale Ausrichtung literarischer Texte auf spezifische Kontexte. Auf der Grundlage eines differenzierten Kontext-Begriffs ${ }^{2}$ werden die untersuchten Texte als Bestandteile der politischen Sphäre verstanden, die einen Teilbereich der reichsstädtischen Kommunikation im 15. Jahrhundert darstellen. Der Fokus liegt dabei auf der ideologischen Ausrichtung bzw. Funktionalisierung, ${ }^{3}$ die - so lautet die These dieses Beitrags - in der Idealisierung der Nürnberger Regierung besteht.

In methodischer Hinsicht lässt sich die Beschreibung einer funktionalen Ausrichtung literarischer Texte auf einen konkreten Diskurszusammenhang nicht allein durch ein literaturwissenschaftliches Begriffsrepertoire im engeren Sinne leisten, sondern erfordert den Rückgriff auf sozialwissenschaftliche Kategorien und Begriffe. Durch Rekurs auf diese können die Formen der kontextuellen Einbindung der Texte differenziert beschrieben werden. Entsprechend rekurrieren die folgenden Überlegungen auf die sozialwissenschaftlichen

1 Auf eine mittlere Ebene des kulturwissenschaftlichen Kontextverständnisses zielen etwa die Überlegungen von Jan-Dirk Müller: Einleitung. In: Ders. (Hg.): Text und Kontext. Fallstudien und theoretische Begründungen einer kulturwissenschaftlich angeleiteten Mediävistik. München 2007, S. VII-XI.

2 Vgl. die genaue Differenzierung des Kontextbegriffs bei Katja Mellmann: Kontext `Gesellschaft«. Literarische Kommunikation - Semantik - Strukturgeschichte. In: Journal of Literary Theory 8 (2014), S. 87-117, hier S. 108-110.

3 Dabei geht es hier vor allem um die Ebene des historischen Problembezugs. Vgl. ebd., S. 109-110.

Nina Nowakowski, Magdeburg 
Begriffe der Gouvernementalität und Propaganda, wobei die Frage, inwiefern diese Begrifflichkeiten das Kriterium der historischen Adäquatheit erfüllen, ausgeblendet bleibt. Im Sinne einer Heuristik sollen die folgenden Überlegungen einen exemplarischen Beitrag zur Suche nach einer Beschreibungssprache leisten, mit der die Funktionalität literarischer Texte im Hinblick auf ihren ১Sitz im Leben` möglichst präzis charakterisiert werden kann.

Im Fokus der Untersuchung stehen die literarischen Darstellungen des städtischen Rats Nürnbergs, da dieser im 15. Jahrhundert als Regierungsinstitution, d. h. als »normativ geregelte, mit gesellschaftlichem Geltungsanspruch dauerhaft strukturierte und über Sinnbezüge legitimierte Wirklichkeit sozialen Handelns «, ${ }^{4}$ das auf die Herrschaftsausübung ausgerichtet war, in der Reichsstadt »die politische Macht allein in den Händen hielt «. ${ }^{5}$ Die Untersuchung soll zeigen, dass Rosenplüt durch eine spezifische Darstellung des Regierungshandelns auf die Legitimation der Ratsherrschaft hinwirkt: Nürnbergs Regierung, die in einem hohen Maße von autokratischen Tendenzen geprägt war, ${ }^{6}$ wird in den Texten als politische Institution dargestellt, die sich um das Wohlergehen der Nürnberger Bevölkerung bemüht. Das politische Handeln des Rats bzw. der Ratsherren wird gerade nicht als autoritär-herrschaftliches Regierungshandeln, sondern in je unterschiedlichen Formierungen der Unterstützung für die Stadtbewohner entworfen.

Der Beitrag knüpft damit an Untersuchungen zur Verbindung zwischen Politik und Literatur in Nürnberg für das 15. Jahrhundert an, die bereits die Relevanz des Rats als einflussreichen politischen Akteur akzentuiert haben. ${ }^{7}$ Insofern der Rat als Gegenstand literarischer Texte bislang allerdings kaum explizit in den Blick genommen wurde, können die folgenden Überlegungen die Perspektive erweitern: Die Fastnachtspiele Rosenplüts, in denen Mitglieder des städtischen Rats auftreten, werden von der Forschung nämlich zumeist nicht

4 Roger Häußling: Institution. In: Johannes Kopp, Anja Steinbach (Hg.): Grundbegriffe der Soziologie. 11. Aufl. Wiesbaden 2016, S. 140-143, hier S. 140.

5 Jörn Reichel: Der Spruchdichter Hans Rosenplüt. Literatur und Leben im spätmittelalterlichen Nürnberg. Stuttgart 1985, S. 113. Die Geschichte des Nürnberger Rats arbeitet detailliert auf Peter Fleischmann: Rat und Patriziat in Nürnberg. Die Herrschaft der Ratsgeschlechter in der Reichsstadt Nürnberg vom 13. bis zum 18. Jahrhundert. Neustadt an der Aisch 2008.

6 Dies beschreibt aus literaturwissenschaftlicher Sicht etwa Werner Williams-Krapp: Literatur in der Stadt: Nürnberg und Augsburg im 15. Jahrhundert. In: Ders. (Hg.): Geistliche Literatur des späten Mittelalters. Kleine Schriften. Tübingen 2012, S. 35-48, hier S. 37-38.

7 Vgl. Eckehard Simon: Die Anfänge des weltlichen deutschen Schauspiels 1370-1530. Untersuchung und Dokumentation. Tübingen 2003, S. 291-348. 
den Fastnachtspielen mit politischer Thematik zugeordnet, ${ }^{8}$ sondern als Spiele mit juridischer Thematik verstanden und als >Gerichtsspiele ‘ klassifiziert. ${ }^{9} \mathrm{Al}-$ lerdings lässt sich im Hinblick auf die Darstellung des Stadtrats zeigen, dass dieser nicht nur als juridische, sondern durchaus auch als politische Instanz in den Fastnachtspielen thematisch wird. Dabei sind die von Rosenplüt gewählten Register bei der Darstellung der Regierung von besonderem Interesse, denn der Stadtrat wird mit Regierungsverfahren in Verbindung gebracht, die mit Michel Foucault als Formen gouvernementalen Handelns beschrieben werden können:

Mit diesem Wort `Gouvernementalität ` ist dreierlei gemeint. Unter Gouvernementalität
verstehe ich die Gesamtheit, gebildet aus den Institutionen, den Verfahren, Analysen und
Reflexionen, den Berechnungen und den Taktiken, die es gestatten, diese recht spezifi-
sche und doch komplexe Form der Macht auszuüben, die als Hauptzielscheibe die Bevöl-
kerung, als Hauptwissensform die politische Ökonomie und als wesentliches technisches
Instrument die Sicherheitsdispositive hat. Zweitens verstehe ich unter >Gouvernementali-
tät` die Tendenz oder die Kraftlinie, die im gesamten Abendland unablässig und seit sehr
langer Zeit zur Vorrangstellung dieses Machttypus, den man als >Regierung` bezeichnen
kann, gegenüber allen anderen - Souveränität, Disziplin - geführt und die Entwicklung
einer ganzen Reihe spezifischer Regierungsapparate einerseits und einer ganzen Reihe
von Wissensformen andererseits zur Folge gehabt hat. Schließlich glaube ich, dass man
unter Gouvernementalität den Vorgang oder eher das Ergebnis des Vorgangs verstehen
sollte, durch den der Gerechtigkeitsstaat des Mittelalters, der im 15. und 16. Jahrhundert
zum Verwaltungsstaat geworden ist, sich Schritt für Schritt `gouvernementalisiert ‘ hat.

8 Vgl. die Aufzählung der politischen Fastnachtspiele Rosenplüts bei Brigitte Stuplich: Das ist dem adel ain große schant. Zu Rosenplüts politischen Fastnachtspielen. In: Jürgen Jaehrling, Uwe Meves, Erika Timm (Hg.): Röllwagenbüchlein. FS Walter Röll. Tübingen 2002, S. 165-186, hier S. 166 (Anm. 7). Eine Ausnahme bildet Des Turken vastnachtspil (K 39).

9 Ingeborg Glier: Rosenplütsche Fastnachtspiele. In: ${ }^{2}$ VL 8 (1990), Sp. 211-232, hier Sp. 220-222. Grundlegendes zu den Gerichtsspielen erarbeitet Rebekka Nöcker: Zur Darstellung von Juristen im frühen Nürnberger Fastnachtspiel. In: Klaus Ridder (Hg.): Fastnachtspiele. Weltliches Schauspiel in kulturellen Kontexten. Tübingen 2009, S. 239-283. Eine sinnvolle Differenzierung zwischen Prozessspielen und Spielen der Rat-Erfragungen schlägt vor Hansjürgen Linke: Aspekte der Wirklichkeits-Wahrnehmung im weltlichen deutschen Schauspiel des Mittelalters. In: Klaus Ridder (Hg.): Fastnachtspiele. Weltliches Schauspiel in literarischen und kulturellen Kontexten. Tübingen 2009, S. 11-61, hier S. 43-44.

10 Michel Foucault: Dits et Écrits. Schriften. Bd III. Frankfurt a.M. 2003, S. 820-821. Dass die spätmittelalterlichen Städte Entwicklungen des modernen Verwaltungsstaats vorweggenommen haben, wurde vielfach beschrieben. Die differenzierten Verwaltungsstrukturen Nürnbergs im 15. Jahrhundert sind maßgeblich an die Institution des Rats gebunden. Vgl. zusammenfassend Eberhard Isenmann: Die deutsche Stadt im Mittelalter 1150-1550. Stadtgestalt, Recht, Verfassung, Stadtregiment, Kirche, Gesellschaft, Wirtschaft. Köln 2012, S. 448-449. 
Es geht Foucault bei der Beschreibung der Gouvernementalität um Praktiken bzw. Denk- und Handlungsweisen des Regierens, ${ }^{11}$ die sich aus verschiedenen Techniken speisen und eher keinen direktiv-autoritären, sondern einen appellativ-solidarischen Charakter aufweisen, um Menschen und menschliche Gesellschaften sindirekt $>\mathrm{zu}$ leiten und zu lenken (vgl. lat. gubernare).

Herausgearbeitet werden soll im Folgenden mit Blick auf Fastnachtspiele Rosenplüts, dass das politische Handeln des Nürnberger Rats vornehmlich nicht durch Praktiken und Maßnahmen der Disziplinierung geprägt ist, sondern als konsiliarisches Handeln erscheint, das an den Bedürfnissen der Bevölkerung orientiert ist. Insofern er als nahbarer Ansprechpartner gezeichnet wird, wirken die Texte auf ein positives Image des Rats hin. Dabei wird ein vermeintlicher Einblick in den Ablauf der Regierungsarbeit gewährt, wobei das entworfene Vorgehen des Rats kaum der historischen Realität entspricht: Die Nürnberger Bevölkerung hatte im 15. Jahrhundert keine Möglichkeit, mit den Regierungsgeschäften der oligarchischen Oberschicht ${ }^{12}$ in Berührung zu kommen, denn es gab z. B. keine persönlichen Vorsprachen beim Rat. ${ }^{13}$ Die Ratsgänge basierten allein auf schriftlichen Eingaben. Die Bevölkerung war "gänzlich von Meinungsfindung und Entscheidungsbildung ausgeschlossen «. ${ }^{14}$ Neben den Mitgliedern des Rats, die sich aus einem sehr engen, durch das Tanzstatut von 1521 festgelegten, ${ }^{15}$ elitären Kreis von Nürnberger Bürgerfamilien rekrutierten, hatten nur ausgewählte Personen Zugang zur Ratsstube. Die personale und räumliche Abschottung der Regierung trug dazu bei, dass die Ratsfamilien bzw. -geschlechter eine Herrschaftsschicht ausbilden konnten, die unter sich blieb. ${ }^{16}$ Die Beteiligung an politischen Prozessen war aufgrund der Ratsverfassung nur wenigen Familien möglich und die Ratsmitglieder wurden durch die jährlichen Ratswahlen fast immer bestätigt, sodass die patrizische Regierung durch Exklusivität und Kontinuität geprägt war. Durch die Organisation des

11 Vgl. Thomas Lemke: Gouvernementalität. In: Clemens Kammler, Rolf Parr, Ulrich Johannes Schneider (Hg.): Foucault-Handbuch. Leben - Werk - Wirkung. Berlin, Heidelberg 2014, S. 260-263, hier S. 261-262.

12 Vgl. Peter Fleischmann: Professionalisierung oder Ausschluß von Führungsschichten in der Reichsstadt Nürnberg? In: Günther Schulz (Hg.): Sozialer Aufstieg. Funktionseliten im Spätmittelalter und in der frühen Neuzeit. München 2002, S. 49-71, hier S. 50.

13 Vgl. Fleischmann: Rat und Patriziat (s. Anm. 5), S. 180.

14 Ebd., S. 307.

15 Vgl. ebd., S. 222-225; und Simon: Die Anfänge (s. Anm. 7), S. 293.

16 Vgl. Reichel: Der Spruchdichter (s. Anm. 5), S. 113. 
Rats wurde die Macht zusätzlich ıverdichtet`: Die Regierungsgeschäfte oblagen dem Kleineren Rat. ${ }^{17}$ Alle wichtigen politischen Entscheidungen wurden ab Ende des 14. Jahrhunderts aber von den sieben Älteren Herren bzw. Septemvirn getroffen, die bis ins 18. Jahrhundert »die eigentlichen Regenten Nürnbergs « ${ }^{18}$ darstellten. Unter ihnen wurden auch im Geheimen Absprachen getroffen, bevor der Kleinere Rat verständigt wurde. ${ }^{19}$ Nürnbergs Regierung konnte gerade auch durch diese Konzentration der politischen Macht auf wenige Akteure das »Stadtleben mit einer an Kontrollsucht grenzenden Beflissenheit ${ }^{20}{ }^{2}$ überwachen.

In der literaturwissenschaftlichen Forschung wurde beschrieben, dass die Nürnberger Fastnachtspiele sich mit der Ordnungspolitik des städtischen Rats bevorzugt im Modus der Verkehrung auseinandersetzen. ${ }^{21}$ Nicht abschließend geklärt ist, wie fastnächtliche Verkehrungen im Hinblick auf die Text-KontextRelationen genau zu denken sind, d. h. ob mit diesen eine grundlegende, die Herrschaftsstrukturen destabilisierende Perspektive einhergeht oder aber in der Begrenztheit der fastnächtlichen Aktivitäten zielgerichtete Transgressionen in einem rituellen Rahmen stattfinden, denen letztlich eine stabilisierende Funktion zukommt. ${ }^{22}$ Mit der Verkehrung ist, darin scheint sich die Forschung weitgehend einig, allerdings die Möglichkeit zur Subversion bestehender Machtverhältnisse und Ordnungsmuster verbunden. Der vorliegende Beitrag setzt an einer anderen Stelle an, indem er im Hinblick auf die Darstellung des Rats zeigt, dass Politik in den Fastnachtspielen keineswegs nur im Modus der Verkehrung thematisiert

$17 \mathrm{Zu}$ den Geschäftsgängen im Kleineren Rat vgl. Fleischmann: Rat und Patriziat (s. Anm. 5), S. $175-186$.

18 Ebd., S. 62.

19 Vgl. ebd.

20 Simon: Die Anfänge (s. Anm. 7), S. 293.

21 Vgl. Klaus Ridder: Fastnachtstheater. Städtische Ordnung und fastnächtliche Verkehrung. In: Ders. (Hg.): Fastnachtspiele. Weltliches Schauspiel in literarischen und kulturellen Kontexten. Tübingen 2009, S. 65-81, hier S. 71.

22 Dieses Problem erörtert ausführlich, wobei er zu einem vorsichtigen Umgang mit dem Begriff der Verkehrung rät, Gerhard Wolf: Komische Inszenierung und Diskursvielfalt im geistlichen und im weltlichen Spiel (Das `Erlauer Osterspiel und die Nürnberger Arztspiele K 82 und K 6). In: Klaus Ridder (Hg.): Fastnachtspiele. Weltliches Schauspiel in literarischen und kulturellen Kontexten. Tübingen 2009, S. 301-326, hier S. 325. Grundlegende Überlegungen zur fastnächtlichen Verkehrung finden sich bei Hedda Ragotzky: Der Bauer in der Narrenrolle. Zur Funktion >verkehrter Welt` im frühen Nürnberger Fastnachtspiel. In: Horst Wenzel (Hg.): Typus und Individualität im Mittelalter. München 1983, S. 77-101; und Werner Röcke: Literarische Gegenwelten. Fastnachtspiele und karnevaleske Festkultur. In: Werner Röcke, Marina Münkler (Hg.): Die Literatur im Übergang vom Mittelalter zur Neuzeit. München, Wien 2004 (Hansers Sozialgeschichte der deutschen Literatur 1), S. 420-445. 
worden ist. ${ }^{23}$ Bei der Darstellung des Nürnberger Rats und auch von Ratgebergremien anderer Regierungen in Rosenplüts Fastnachtspielen wird vielmehr eine ratskonforme Haltung ${ }^{24}$ zum Ausdruck gebracht und aktiv politische Meinungsbildung betrieben. Rosenplüts Haltung der Nürnberger Regierung gegenüber soll dabei nicht pauschal als reaktionär charakterisiert werden, ${ }^{25}$ sondern die Darstellungen des Rats sollen im Hinblick auf Funktionslogiken vielmehr möglichst differenziert beschrieben werden. Dabei wird zu zeigen sein, dass das Regierungshandeln des städtischen Rats in den Spielen als am Wohlergehen der Stadtbewohner orientiert, als persönliche Auseinandersetzung mit der Bevölkerung und als anderen Regierungsformen überlegen dargestellt wird.

Die Texte erscheinen in recht unmittelbarer Weise in den politischen Kommunikationszusammenhang der Reichsstadt eingebunden, insofern sie sich im Sinne einer PR-Kampagne für den Stadtrat bzw. als literarische Propaganda verstehen lassen, mit der versucht wird, „einen bestimmten Adressatenkreis durch Informationslenkung für eigennützige Zwecke $\mathrm{zu}$ gewinnen und diese Zwecke zugleich zu verschleiern «. ${ }^{26}$ Ziel der literarischen Darstellung des Rats ist es, die Relation der Bevölkerung zur Institution zu beeinflussen. ${ }^{27}$ Rosenplüt stellt das Regierungshandeln des Stadtrats in einer bestimmten Weise dar und wirkt in seinen Texten damit auf die Legitimation, den Erhalt und die Ausbreitung (vgl. lat. propagare) der Macht des Rats hin. ${ }^{28}$ Doch indem die Vorteile akzentuiert werden, die sich durch das gouvernementale Handeln der Regierung für die Nürnberger Bevölkerung ergeben, wird diese Ausrichtung verschleiert.

23 Dies deutet Ridder: Fastnachtstheater (s. Anm. 21), S. 72, an.

24 Inwiefern Rosenplüts Texte die Politik des Rats distanziert-kritisch oder aber konformistisch darstellen, diskutiert Reichel: Der Spruchdichter (s. Anm. 5), S. 211-220.

25 Dies kritisiert Carla Meyer: Wie und warum wird städtische Identität zum Thema? Nürnberg im Städtelob um 1500. In: Dies., Christoph Hartmann (Hg.): Identität und Krise? Zur Deutung vormoderner Selbst-, Welt- und Fremderfahrungen. Münster 2007, S. 119-136, hier S. 134. Etwas differenzierter sieht das Reichel: Der Spruchdichter (s. Anm. 5), S. 158-168 und S. 211-220.

26 Sabine Doering-Manteuffel: Propaganda. In: Gerd Ueding (Hg.): Historisches Wörterbuch der Rhetorik. Bd. 7. Tübingen 2005, Sp. 266-283, hier Sp. 267. Jörn Reichel hingegen geht davon aus, dass Rosenplüts Texte von einem kritischeren Verhältnis zum Rat geprägt sind. Vgl. Reichel: Der Spruchdichter (s. Anm. 5), S. 193.

27 Vgl. Edward L. Bernays: Propaganda. New York 1928, S. 25.

28 Vgl. Fleischmann: Rat und Patriziat (s. Anm. 5), S. 311. 


\title{
2 Der Nürnberger Rat als guter Hirte: Wider die Partikularisierung
}

Die folgenden Überlegungen werden sich vor allem auf die Fastnachtspiele Rosenplüts konzentrieren, doch vorangestellt ist ihnen die Analyse eines Textausschnitts aus Rosenplüts Lobspruch auf Nürnberg ${ }^{29}$ von 1447, der als Städtelob einer rhetorisch-politischen Textsorte zuzuordnen ist und deshalb programmatische Aussagen macht, wobei auch der reichsstädtische Rat bedacht wird:

\begin{abstract}
Noch vind ich ein dingk in Nurmberg,
Das ist das allerweißlichst wergk,

Das ich in keiner stat nie vant:

Gesetz und ordenung wirt oft zutrant,

Wo man mer hirten hat dann ein.

[...]

In Nurmberg ist newr ein hirt,

Der nie mit dem vihe hat gestolzirt

Und also getreulich zugehutt hat

Der großen hert, der ganzen stat,

Das sie kein unziffer nie mocht vergiften,

Das oft geschicht auf hohen stiften

Und auch wo einfeltig zunft sein.

Do sehet der teufel seinen samen ein,

Das mordt und jamer oft wirt awßgebrütt.

Bei vil hirten wirt oft ubel gehutt.

Der hirte ist der weise, fursichtig rat,

Der nacht und tag, fru und spat

Getreulich hut aller gelerten und leien.

Wo einer sich mit dem andern wil zweien,

So hutt der hirte so weißlich zu,

Das sie noch bleiben bei sollicher ru,

Das sie keins hirten mer begern.
\end{abstract}

$$
\text { (V. 347-V. 371) }
$$

Der Rat wird im Lobspruch auf Nürnberg, der so breit wie kein anderer Text Rosenplüts überliefert ist, ${ }^{30}$ als guter Hirte entworfen und damit nicht als eine

29 Im Folgenden zitiert nach: Hans Rosenplüt: Reimpaarsprüche und Lieder. Hg. von Jörn Reichel. Tübingen 1990, S. 220-234.

30 Zur Überlieferung vgl. Reichel: Der Spruchdichter (s. Anm. 5), S. 253-254. Die Frage, ob Rosenplüt den Text aus persönlichem Kalkül geschrieben hat, um eine Verlängerung seines Vertrags als Büchsenmeister beim Rat zu erreichen, ist umstritten. Vgl. ebd., S. 201; und Matthias Kirchhoff: Gedächtnis in Nürnberger Texten des 15. Jahrhunderts. Gedenkbücher Brüderbücher - Städtelob - Chroniken. Nürnberg 2009, S. 226. 
herrschende Institution, sondern als Instanz gezeigt, die ihrer Herde - der Nürnberger Bevölkerung - ein friedvolles, sicheres und gutes Leben garantiert. Die Aufgabe des Hirten ist das Hüten, also das Zusammenhalten und Schützen der Herde. Insofern hier das »Heil der Herde [...] das wesentliche Zielobjekt ${ }^{31}$ des Handelns des Rats darstellt, impliziert Rosenplüt eine pastoral-gouvernementale Idee des Regierens. Indem der weise, fursichtig rat als Hirte entworfen wird, wird ihm eine Souveränität zugesprochen, die sich von anderen Formen der Souveränität durch ihre Funktion und ihre Alleinstellung unterscheidet. ${ }^{32}$ Der Hirte kontrolliert die Herde nicht, um Kontrolle auszuüben, sondern um diese zu schützen. Dabei kann es nur einen Hirten geben, ${ }^{33}$ denn dort, wo vil hirten wirken, d. h. wo die Macht dezentralisiert ist, wirt oft ubel gehutt. Partikularisierung wird als Bedrohung entworfen, ${ }^{34}$ Einheitlichkeit und Geschlossenheit bei Hirte und Herde werden als Garanten für Sicherheit und sozialen Frieden dargestellt: ${ }^{35}$ Der Rat, der in der Realität aus einer Gruppe verschiedener Vertreter besteht, wird hier als eine im christlichen Verständnis durchweg positiv besetzte Instan $z^{36}$ personifiziert und damit als ein einzelner politischer Akteur entworfen, der Verantwortung für das Heil der Bevölkerung übernimmt. ${ }^{37}$ Zugleich wird der Rat als Instanz dargestellt, die einer Partikularisierung entgegensteht: Erwähnt wird, dass der Rat als Hirte hut aller gelerten und leien, d. h. seine Aufgabe richtet sich auf alle Schäflein. Auch die Verantwortung des christlichen pastor, der hier als Modell dient, erstreckt sich, das sagen schon die Kirchväter, auf das Heil aller. ${ }^{38}$ Als Herde bilden die Nürnberger eine Einheit, entsprechend sollen sie sich nicht zweien. Dass der Rat seine Herde besonders gut ruhig halten kann (vgl. V. 370), hängt damit zusammen, dass Partikularisierung nicht nur ein Ausschlusskriterium für die Regierten, sondern auch das Regieren

31 Michel Foucault: Geschichte der Gouvernementalität I. Sicherheit, Territorium, Bevölkerung. Frankfurt a.M. 2004, S. 189.

32 Vgl. Kirchhoff: Gedächtnis (s. Anm. 30), S. 224.

33 Vgl. Foucault: Geschichte der Gouvernementalität (s. Anm. 31), S. 211-212.

34 Rosenplüts antipartikulares Interesse betont Reichel: Der Spruchdichter (s. Anm. 5), S. 223.

35 Vgl. zur Akzentuierung, dass Übereinstimmung zwischen Rat und Bevölkerung herrsche, Meyer: Städtische Identität (s. Anm. 25), S. 127-128.

36 Zum Pastorat als christlicher Matrix vgl. Foucault: Geschichte der Gouvernementalität (s. Anm. 31), S. 217-277.

37 Zum Prinzip der Verantwortlichkeit, das die pastorale Machtausübung prägt, vgl. Michael Ruoff: Foucault-Lexikon. Entwicklung - Kernbegriffe - Zusammenhänge. Paderborn 2007, S. 162.

$38 \mathrm{Zu}$ diesem Aspekt u. a. bei Johannes Chrysostomos, in der Benediktsregel und in der regula pastoralis von Gregor d. Gr. vgl. Foucault: Geschichte der Gouvernementalität (s. Anm. 31), S. 246-247. 
darstellt. So bündeln sich beim Rat etwa Legislative und Exekutive bzw. Gesetz und ordenung. Die Dimensionen der Wohltätigkeit und der Sorge um die Herde lenken nicht nur vom Beunruhigenden der zentralistisch organisierten Machtausübung ab, ${ }^{39}$ sondern basieren, so wird hier nahegelegt, gerade auf der Zentralisierung und Monopolisierung der Macht des Rats: Aufgrund seiner Alleinstellung kann der Hirte seine pflegerische Verantwortung übernehmen, dem Gedeihen, der Versorgung und der Gesundheit der Herde dienen. Der Gehorsam ${ }^{40}$ gegenüber dieser Institution erscheint angebracht, weil ihr Handeln am Gemeinwohl ausgerichtet ist. ${ }^{41}$ Im Lobspruch auf Nürnberg wird akzentuiert, wie wichtig und politisch sinnvoll die Bündelung der Macht beim Rat ist, indem diese mit der Übernahme von pastoraler Verantwortung kurzgeschlossen wird.

Dass der Rat, der sich aus verschiedenen Mitgliedern bzw. Ratsherren zusammensetzt, tatsächlich im Sinne eines Ideals der Einheit agiert, wie die Hirten-Personifikation im Lobspruch nahelegt, wird von Rosenplüt im Kontext des Fastnachtspiels Der vastnacht und vasten recht (K 72) ${ }^{42}$ ausgestellt. In diesem Spiel wird die Fastnacht von der Fastenzeit verklagt, weil diese sie verdrängt habe, wodurch sie großen schaden genumen (S. 624, V. 9) habe. In dem Reihenspiel äußern sich zunächst die Klägerin, die Fastenzeit, dann die Beklagte, die Fastnacht, und schließlich kommen nacheinander neun Ratsherren zu Wort. Die Ratsherren verhandeln nicht darüber, ob nun die Fastnacht oder aber die Fastenzeit im Recht sei, denn obwohl sie in einem juridischen setting agieren, wollen sie gar kein Urteil fällen. ${ }^{43}$ Dies wird schon durch die Rede des ersten Ratsherrn verdeutlicht:

\footnotetext{
Der erst ratherr:

Ich hab die Fasnacht und die Fast wol vernumen,

Wie sie paide für recht sein kumen.

Wir künnen in hart sprechen ain recht,

Das sie paide bedunkt schlecht.
}

39 Vgl. ebd., S. 191.

40 Dass mit dem Pastorat im Christentum eine besondere Form der Machtausübung durch Gehorsam entsteht, wird betont ebd., S. 254-267.

41 Dass hier das Ideal des gemeinen Nutzens zugrunde liegt, betont Kirchhoff: Gedächtnis (s. Anm. 30), S. 227.

42 Im Folgenden zitiert nach: Fastnachtspiele aus dem fünfzehnten Jahrhundert. Bd. 2. Hg. von Adelbert von Keller. Stuttgart 1853, S. 624-627. Die Zählung nach von Keller (K 1, K 2 etc.) wird in Klammern hinter den Titeln der berücksichtigten Spiele angegeben.

43 Schon Eckehard Catholy bemerkt, dass die Gerichtsspiele eine Tendenz zur »Entscheidungslosigkeit« (Eckehard Catholy: Das Fastnachtspiel des Spätmittelalters. Gestalt und Funktion. Tübingen 1961, S. 198) aufweisen. 
Es muß ie ir ains unterligen.

Mich dunkt das pest der urteil iezund geswigen.

(S. 625, V. 13-19)

Der erste Ratsherr will das urtail sechs wochen (S. 625, V. 20) aufschieben und auch die anderen Ratsherren wollen des meien damit erpeiten (S. 626, V. 3), also die Entscheidung auf einen Zeitpunkt vertagen, zu dem die Fastenzeit definitiv vorbei ist. Der letzte Ratsherr äußert sich entsprechend salomonisch:

Der neunt ratherr:

Ich hab gelernt in meiner jugent,

Auß einer not mach ich ain tugent.

Wir wollen die Fasten gern ern,

Die kan uns von narrhait kern

Und damit die Fasnacht nit übergeben,

Sie möcht uns wider kumen eben.

(S. 627, V. 5-11)

In der Forschung wurde vor allem die Komik betont, die aus der Unfähigkeit der Ratsherren erwachse, ein Urteil zu fällen. ${ }^{44}$ Indem die Ratsherren nicht urteilen und damit den $»$ Rechtsstreit aus den Angeln $\aleph^{45}$ heben, umgehen sie eine klare Positionierung, was politisches Geschick im Hinblick auf unterschiedliche Interessensgruppen nahelegt. Diese werden hier durch die Personifikationen in besonders deutlichem Kontrast gezeichnet. Doch der Rat vermeidet bei allen prinzipiellen Gegensätzen, die mit der Fastenzeit und der Fastnacht verbunden sind, dass eine Partei unterliegt. Es geht darum, die Integration unterschiedlicher Interessen als Ziel des Rats zu verdeutlichen. So rückt die juridische Thematik gegenüber der Darstellung des Rats als politischem Akteur, der einvernehmlich darauf zielt, diplomatisch zu vermitteln, in den Hintergrund. Das Spiel entwirft den Rat als im Meinen und im Handeln homogene Gruppe von Ratsherren, die in der Rolle von Mediatoren auch bei gegenläufigen Interessen für Ruhe bzw. sozialen Frieden in der Stadt sorgen. ${ }^{46}$

44 Vgl. Nöcker: Darstellung von Juristen (s. Anm. 9), S. 264-265 und 270-271.

45 Hedda Ragotzky und Christa Ortmann: Itlicher zeit tut man ir recht. Zu Recht und Funktion der Fastnacht aus der Sicht Nürnberger Spiele des 15. Jahrhunderts. In: Hans-Joachim Ziegeler (Hg.): Ritual und Inszenierung. Geistliches und weltliches Drama des Mittelalters und der Frühen Neuzeit. Tübingen 2004, S. 207-218, hier S. 212. Die Analyse bezieht sich allerdings auf Das spil von der vasnacht und vasten recht (K 73), das thematisch jedoch eng mit Der vastnacht und vasten recht (K 72) verwandt ist.

46 Dass der soziale Frieden im Lobspruch auf Nürnberg besonders akzentuiert wird, betont Kirchhoff: Gedächtnis (s. Anm. 30), S. 227. 


\section{Von der Rechtsprechung zur Beratung: Bürgernähe statt law and order}

Besonders an den Bedürfnissen der reichsstädtischen Bevölkerung interessiert erscheinen die Vertreter des Nürnberger Rats im Fastnachtspiel Der wittwen und tochter vasnacht (K 97). ${ }^{47}$ Hier treten die titelgebenden Figuren vor einem Richter zusammen und erläutern ihre Absicht, beide heiraten zu wollen. Man wolle herausfinden, welche dies vor der anderen tun solle bzw. dürfe. Der Text ist vor dem Hintergrund der vielfältigen Regelungen rund um die Eheschließung zu verorten, die der Nürnberger Rat vornahm. Diese liegen in Form von Hochzeitsverordnungen vor, ${ }^{48}$ die eine große Anzahl von Aspekten rund um Heiratsanbahnung, Hochzeitsfestivitäten usw. sehr detailliert festlegen. Die darin sich ausdrückende »exzessive Ordnungspolitik « ${ }^{49}$ der reichsstädtischen Regierung wird von Rosenplüt in Der wittwen und tochter vasnacht entschärft, indem die Ratsherren als bürgernahe Ansprechpartner in Fragen der Eheschließung dargestellt werden. Die Ausgangssituation der Spielhandlung scheint eine parodistische Verkehrung des >Kontrollwahns der Regierung im Hinblick auf Eheschließungen nahezulegen, denn der dargestellte Fall offenbart, dass bei allen Bemühungen, genau zu regeln, wer unter welchen Umständen wie heiraten darf, dennoch keine lückenlose Kontrolle möglich ist. Auch wenn genau festgelegt ist, wann eine junge Frau heiratsfähig ist und wann eine Witwe erneut heiraten kann, wird durch die Koinzidenz des heiratswilligen Mutter-Tochter-Paares deutlich, dass in der Praxis durchaus Unklarheiten auftreten können. Im vorliegenden Fall ist nicht klar, wer das Vorrecht auf eine Eheschließung besitzt. Im Gegensatz zu den abstrakt-überindividuellen Vorschriften, durch die eherechtliche Belange in Nürnberg geregelt werden, wird der Rat im Spiel als zugängliche Instanz präsentiert, die für die Lücke im Eherecht ohne viel Aufhebens und ganz sunbürokratisch findet. Der Stadtrat tritt dabei nicht als Behörde in Erscheinung, sondern als Gremium, das beratend agiert.

Nachdem die Frauen - explizit ohne fürsprechen (S. 746, V. 10) ${ }^{50}$ - ihr Anliegen vorgebracht haben, ergeht vom Bürgermeister, der hier entsprechend des

47 Im Folgenden zitiert nach: Fastnachtspiele aus dem fünfzehnten Jahrhundert. Bd. 2. Hg. von Adelbert von Keller. Stuttgart 1853, S. 746-750.

48 Vgl. August Jegel: Altnürnberger Hochzeitsbrauch und Eherecht, besonders bis zum Ausgang des 16. Jahrhunderts. In: Mitteilungen des Vereins für Geschichte der Stadt Nürnberg 44 (1953), S. 238-274.

49 Ridder: Fastnachtstheater (s. Anm. 21), S. 71.

50 Vgl. Nöcker: Darstellung von Juristen (s. Anm. 9), S. 277. 
juridischen settings die Funktion des Richters übernimmt, ${ }^{51}$ folgende Aufforderung an den städtischen Rat:

Der richter:

Ir herrn, ir habt sie paid wol vernumen, Warüm sie für ainn rat sein kumen.

Die muter ist nach mannen geitig,

So maint di tochter, sie sei auch zeitig.

$\mathrm{Nu}$ gebt in paiden ain unterscheid,

Welche an dem letzten peit,

Wann sie paid heten gern man.

(S. 747, V. 16-23)

In einer Ratssitzung ${ }^{52}$ äußern nun die Ratsherren der Reihe nach ihre Meinung zu dem Fall, wobei immerhin acht der zehn Ratsherren dazu raten, zunächst die Tochter zu verheiraten. ${ }^{53}$ Von der Forschung wurden die Obszönitäten akzentuiert, ${ }^{54}$ welche die Redepartien prägen. Die verschiedenen topischmisogynen Verweise auf die weibliche Unersättlichkeit sollen die Notwendigkeit der Eheschließung für beide Frauen verdeutlichen. So scheint diese als Ziel, die Frage der Reihenfolge ist hingegen nicht entscheidend. Die Witwe erkennt das an und zeigt sich als gut beratene Städterin:

Di witwe:

Herr der bürgermaister, ich dank euch allen.

Ir habt uns paiden wol gevallen

Und habt uns recht und wol entschaiden.

$\mathrm{Nu}$ wil ich trachten umb ainn aiden

Und wil meiner tochter ainn jungen man geben.

(S. 750, V. 13-18)

Hier kommt es nicht auf fastnächtliche Verkehrung, sondern auf die Lösungsorientiertheit des Rats an, der den Streit durch Beratung in Einigkeit überführt.

Das Spiel weist thematische Parallelen zum Spiel Der jüngling, der ain weip nemen wil (K 41) ${ }^{55}$ auf, in dem die Frage verhandelt wird, wann ein Mann sich im heiratsfähigen Alter befindet. Durch einen jungen und heiratswilligen Mann

51 Vgl. ebd., S. 276-278; und Linke: Aspekte der Wirklichkeits-Wahrnehmung (s. Anm. 9), S. 43.

52 Vgl. Nöcker: Darstellung von Juristen (s. Anm. 9), S. 277.

53 Vgl. Linke: Aspekte der Wirklichkeits-Wahrnehmung (s. Anm. 9), S. 43.

54 Vgl. ebd., S. 43-44; und Elisabeth Keller: Die Darstellung der Frau in Fastnachtspiel und Spruchdichtung von Hans Rosenplüt und Hans Folz. Frankfurt a.M. u. a. 1992, S. 61-62.

55 Im Folgenden zitiert nach: Fastnachtspiele aus dem fünfzehnten Jahrhundert. Bd. 1. Hg. von Adelbert von Keller. Stuttgart 1853, S. 314-319. 
veranlasst, stellt diese Frage ein richter (S. 315, V. 3) nach Auskunft der Edition Kellers einer Reihe von juristen (S. 315, V. 4) bzw. doctores, die sie ringsum erörtern. ${ }^{56}$ Aber in einer Handschrift werden diese als ratherrn (vgl. Cgm 714, fol. $417 \mathrm{v}-420 \mathrm{v}^{57}$ ) bezeichnet. Und wie in Der wittwen und tochter vasnacht kommt es auch hier gerade auf die individuelle und persönliche Beratung des jungen Mannes durch den Rat an, ${ }^{58}$ durch die der Jüngling letztlich zu dem Schluss kommt, dass es für ihn noch nit zeit (S. 319, V. 11) sei, sich eine Ehefrau zu suchen. Der reichsstädtische Rat, so scheinen diese beiden »Spiele der RatErfragungen ${ }^{59}$ nahezulegen, kümmert sich nicht nur mittels abstrakter Vorschriften um Fragen der Eheschließung, sondern hilft der Stadtbevölkerung, indem er ihr beratend zur Seite steht. Im Rahmen der Rechtsprechung, die in Nürnberg auch in den Händen des Rats lag, ${ }^{60}$ ist anders als bei den Ratssitzungen die Begegnung und Kommunikation zwischen der Bevölkerung und Ratsmitgliedern möglich. Vielleicht erweisen sich auch deshalb Gerichtsspiele als erfolgreicher Typus des Nürnberger Fastnachtspiels: ${ }^{61}$ Hier kann der Rat als nah- und erreichbarer Berater, der den Bewohnerinnen und Bewohnern in Fragen der Lebensführung unterstützend zur Seite steht, entworfen werden. Der dramatische Modus und das juridische setting erlauben es, die unzugänglichautokratisch agierende Regierungsinstitution als persönlichen Kommunikationspartner zu inszenieren, der nicht an der Durchsetzung von Recht und Ordnung, sondern an einer individuellen und unkomplizierten Lösungsfindung interessiert ist und auf konsiliarische Weise seine Regierungsverantwortung wahrnimmt. ${ }^{62}$ Insofern lässt sich die Entscheidungsunwilligkeit, die viele Gerichtsspiele auszeichnet, ${ }^{63}$ funktional auf eine gouvernementale Darstellung der Regierung beziehen. Der Rat erscheint nicht als Souverän, sondern als konsiliarische Instanz. Dabei lässt sich die Beratung als kommunikativer Modus

56 Vgl. Nöcker: Darstellung von Juristen (s. Anm. 9), S. 256-257.

57 München, Staatsbibliothek, Cgm 714. Vgl. das Digitalisat: https://daten.digitale-sammlun gen.de/ db/0002/bsb00024106/images/ (Zugriff am 01.02.2020).

58 Nöcker zufolge handelt es sich um eine »Ratgebersituation« (Nöcker: Darstellung von Juristen [s. Anm. 9], S. 278).

59 Linke: Aspekte der Wirklichkeits-Wahrnehmung (s. Anm. 9), S. 43.

60 Vgl. Rebekka Nöcker: Überlegungen zur Rechtswirklichkeit im frühen Nürnberger Fastnachtspiel. In: LiLi 163 (2011), S. 66-87, hier S. 79-80.

61 Vgl. Ridder: Fastnachtstheater (s. Anm. 21), S. 71.

62 Nöcker sieht richtig, dass Der wittwen und tochter vasnacht (K 97) auf das »Erteilen von Ratschlägen (hier: Eheunterweisung)« (Nöcker: Darstellung von Juristen [s. Anm. 9], S. 252) hinausläuft.

63 Vgl. Catholy: Das Fastnachtspiel (s. Anm. 43), S. 198-205; und Nöcker: Darstellung von Juristen (s. Anm. 9), S. 271. 
des gouvernementalen Handelns verstehen, der nicht auf Disziplinierung, sondern auf Unterstützung setzt.

Die Entwürfe des Stadtrats in den beiden Spielen weisen Ähnlichkeiten mit der in der literaturwissenschaftlichen Mediävistik beschriebenen Funktion von feudalen Beratungsszenen in höfischen Erzählungen auf. Beratungsszenarien gelten aus geschichtswissenschaftlicher Perspektive als zentraler Bestandteil politischer Prozesse in der Vormoderne ${ }^{64}$ Als Grundmuster mittelalterlicher Herrschaftskommunikation und als Funktionsstellen politischer Entscheidungsprozesse fanden diese auch Eingang in die Literatur. ${ }^{65}$ Beratungen bilden vor allem »Kristallisationspunkte für Handlung und Situationsmuster feudaler Epik «. ${ }^{66}$ Dabei hat das Motiv der Beratung eine klare Funktion: Wenn etwa eine Herrscherfigur wie Karl der Große seine Lehnsmänner zum Rat zusammenruft, dient dieser meist dazu, funktionierende Herrschaft zu illustrieren. Der Rat erscheint dabei als topisches Motiv, das die gemeinschaftliche Absicherung von Herrscherhandeln sichtbar macht. Die Darstellung einer Beratung qualifiziert den Herrscher als guten Herrscher. Entsprechend selten wird Beratungshandeln in detaillierter Form dargestellt: »[N]icht der Inhalt des Rats zählt, sondern daß er in öffentlicher Versammlung erteilt wird. « ${ }^{67}$ Für diese Funktionalisierung von Rat sind differenzierte Darstellungen von Beratungsabläufen nicht erforderlich. Eine legitimatorische Funktionalisierung ist auch für die beiden hier untersuchten Fastnachtspiele erkennbar, doch sind die historischen Voraussetzungen dabei andere als in feudalen Kontexten: Der Nürnberger Stadtrat ist eine politische Institution, die nicht das Regierungshandeln eines feudalen Herrschers als »beratende versammlung ${ }^{68}{ }^{6}$ konsiliarisch begleitet, sondern dieses selbst verantwortet. Entsprechend ist die legitimierende Funktion nicht auf eine Herrscherfigur, sondern auf die Institution des Rats selbst gerichtet. Dabei geht es nicht nur um einen Ausweis richtigen Regierens, sondern zudem um die Etablierung einer spezifischen Idealvorstellung von der reichsstädtischen Regierung, die nicht direktiv und autoritär, sondern appellativ und konsiliarisch agiert.

$64 \mathrm{Vgl}$. Gerd Althoff: Colloquium familiare - colloquium secretum - colloquium publicum. Beratung im politischen Leben des früheren Mittelalters. In: Frühmittelalterliche Studien 24 (1990), S. 145-167 (wieder in: Ders.: Spielregeln der Politik im Mittelalter. Kommunikation in Frieden und Fehde. Darmstadt 1997, S. 157-184).

65 Vgl. Doris Ruhe: Ratgeber. Hierarchie und Strategien der Kommunikation. In: Karl-Heinz Spieß (Hg.): Medien der Kommunikation im Mittelalter. Wiesbaden 2003, S. 63-82.

66 Jan-Dirk Müller: Ratgeber und Wissende in heroischer Epik. In: Frühmittelalterliche Studien 27 (1993), S. 124-146, hier S. 124.

67 Müller: Ratgeber (s. Anm. 66), S. 126.

68 Matthias Lexer: Mittelhochdeutsches Handwörterbuch. Bd. 2. Leipzig 1876, Sp. 347. 


\section{Fremde Regierungen: Die Nürnberger Ratsherrschaft als Heilsbringer}

Dass Regierung und Rat wie bei der Nürnberger Ratsherrschaft zusammenfallen, ist nicht in jedem Herrschaftsgefüge gegeben. Allerdings spielt der Rat in Rosenplüts Spielen auch in Bezug auf andere Formen der Herrschaftsausübung eine Rolle. Im Hinblick auf feudal-herrschaftliche Kontexte verdeutlicht Rosenplüt, dass die Beratung von Herrschern entscheidenden Einfluss auf deren politischen Erfolg hat. Im Spiegel anderer Herrschaftsformen wird dabei die Überlegenheit der Nürnberger Ratsherrschaft gegenüber feudalen Herrschaftsordnungen und vor allem dem Reich verdeutlicht. Dies wird in den im Folgenden betrachteten Spielen zum einen durch eine positiv-affirmierende Darstellung eines fremden feudalen Konzils und zum anderen im Modus der Abgrenzung von negativdysfunktionalem Regierungshandeln des Kaisers unter Beteiligung eines beratenden Gremiums illustriert.

Inwiefern feudale Herrscher von ihren Ratgebern unterstützt werden können, macht Des Turken vastnachtspil (K 39) ${ }^{69}$ deutlich. Es handelt sich um das am häufigsten überlieferte Spiel Rosenplüts und ist wenige Jahre nach der Eroberung Konstantinopels im Jahr 1453 entstanden, kann also in einem konkreten politischen Kontext verortet werden. Im Rahmen der Handlung ist der Sultan bzw. Kaiser der Türken mit seinem weisen rat (S. 288, V. 8) der Kritik von Vertretern des Reichs ausgesetzt: Kaiser, Papst und Fürsten sowie deren Boten äußern dem Sultan gegenüber sehr unflätig Beschimpfungen, Vorwürfe und Drohungen. ${ }^{70}$ Dieser und seine Räte setzen sich allerdings zur Wehr und zeigen sich dabei nicht nur rhetorisch überlegen, ${ }^{71}$ sondern üben ihrerseits Kritik an den Missständen im Reich: ${ }^{72}$

69 Im Folgenden zitiert nach: Fastnachtspiele aus dem fünfzehnten Jahrhundert. Bd. 1. Hg. von Adelbert von Keller. Stuttgart 1853, S. 288-304.

70 Vgl. Christiane Ackermann: Dimensionen der Medialität: Die Osmanen im Rosenplütschen >Turken Vasnachtspil« sowie in den Dramen des Hans Sachs und Jakob Ayrer. In: Klaus Ridder (Hg.): Fastnachtspiele. Weltliches Schauspiel in literarischen und kulturellen Kontexten. Tübingen 2009, S. 189-220, hier S. 204-206; und Glenn Ehrstine: Fastnachtsrhetorik. Adelskritik und Alterität in >Des Turken Vasnachtspil«. In: Werkstatt Geschichte 37 (2004), S. 7-23, hier S. 13-15.

71 Vgl. Sebastian Coxon: Weltliches Spiel und Lachen. Überlegungen zur Literarizität, Theatralität und Performativität des Nürnberger Fastnachtspiels des 15. Jahrhunderts. In: Klaus Ridder (Hg.): Fastnachtspiele. Weltliches Schauspiel in literarischen und kulturellen Kontexten. Tübingen 2009, S. 221-238, hier S. 224-225.

72 Vgl. Ridder: Fastnachtstheater (s. Anm. 21), S. 69. 
Der dritt raut des dürgen:

[...]

Ir seit all ungetreu ainander

Und habt bös münz, das ist das ander,

Und falsch richter und ungetreu amptleut.

Wo lebt einer, der ein solchs außreut?

[...]

Und habt pfaffen, die hohe ross reiten,

Die da sölten umb den glauben streiten,

Und böse gericht und untreu herren,

Die müest ir mit eur arbeit neren,

Und habt groß beschwarung und klein frid.

Wo ist einer, der das als abschnid?

Das sol unser fürst als reformiern,

Das hat man gesechen an dem gestirn,

Das eur got in darzuo wil haben,

Das er die übel all sol ab graben,

Und sol euch machen ein rechte reformatzen,

Dar um sült ir in als gering niht schatzen.

(S. 296, V. 4 - S. 297, V. 6)

Die Darstellung der Türken ist in der Forschung treffend als Form der polemischen Idealisierung charakterisiert worden, die im Sinne einer Instrumentalisierung für Nürnberger Belange genutzt werde. ${ }^{73}$ Das Verhältnis zwischen Reichsstädtern und Türken in diesem Text wurde auch als Zweckbündnis beschrieben ${ }^{74}$ und es wurde konstatiert, dass »das Türkenthema als Staffage dient, innere politische und gesellschaftliche Fragen aus reichsstädtischer Perspektive abzuhandeln «. ${ }^{75}$ Der Sultan und seine Ratgeber nehmen eine Stellvertreterfunktion für die Nürnberger ein, insofern ihnen die Kritik der Reichsstädter in den Mund gelegt wird. ${ }^{76}$ Der Zustand des Reichs wird als in verschiedener Hinsicht korrumpiert dargestellt: ${ }^{77}$ Die Richter seien falsch, die Geistlichen wenig am Glauben interessiert, die Lehnsmänner untreu. Die Situation sei von

73 Vgl. Ackermann: Dimensionen der Medialität (s. Anm. 70), S. 209.

74 Vgl. Ehrstine: Fastnachtsrhetorik (s. Anm. 70), S. 22.

75 Michael Schilling: Aspekte des Türkenbildes in Literatur und Publizistik der frühen Neuzeit. In: Wolfgang Harms, Michael Schilling (Hg.): Das illustrierte Flugblatt der frühen Neuzeit. Traditionen, Wirkungen, Kontexte. Stuttgart 2008, S. 227-244, hier S. 236. Vgl. Christiane Ackermann: Von pösen haiden und mahumetischen bluthunden. Die Politisierung des Monsters in der Vormoderne. In: Sabine Kyora, Uwe Schwagmeier (Hg.): How To Make A Monster. Konstruktionen des Monströsen. Würzburg 2011, S. 41-60, hier S. 49-50.

76 Vgl. Ehrstine: Fastnachtsrhetorik (s. Anm. 70), S. 11.

77 Vgl. Ackermann: Dimensionen der Medialität (s. Anm. 70), S. 208; Ragotzky: Der Bauer (s. Anm. 22), S. 95-97. 
Unfrieden geprägt. Zudem wird mit bös münz die problematische Finanzsituation des Kaisers thematisiert. ${ }^{78}$ Dass etwa zur Zeit der Entstehung des Spiels die Nürnberger bei Friedrich III. um finanzielle Unterstützung für die Reichsstädte angefragt hatten (1458), ihnen aber eine Absage erteilt wurde, könnte hierfür eine Erklärung liefern. Der Kaiser hatte finanzielle Probleme und wollte nicht zahlen. ${ }^{79}$ Auf der Handlungsebene verbünden sich die Nürnberger mit den türkischen Besuchern, denen der Stadtrat Geleit zusichert, d. h. Schutz vor Kaiser, Fürsten und Papst gewährt. Anders als die Nürnberger sind die Türken keine Christen und ihr feudales Herrschaftssystem entspricht eher dem des Reichs als dem der Reichsstadt, doch im Gegensatz zum Reich sind die Türken, so soll deutlich werden, nicht von einer inneren Zersetzung ihres Systems betroffen. Rosenplüt ermöglicht in diesem Spiel, »das Heimische durch die Augen und aus der Perspektive des Fremden zu sehen $«{ }^{80}$ Dabei geht es um eine Nobilitierung der heimischen Nürnberger Herrschaftsverhältnisse. Dies zeigt sich u. a. in der Inszenierung des türkischen Rats: Der Sultan und seine Ratgeber erscheinen als vereinte und homogen agierende Gruppe, während das Reich partikularisiert wirkt, ${ }^{81}$ denn dort ist man ungetreu ainander. Die Herrschaftsorganisation der Türken und der Reichsstadt werden parallelisiert und dem als korrumpiert und dysfunktional entworfenen Reich, für das eine rechte reformatzen notwendig erscheint, ${ }^{82}$ gegenübergestellt. Die Türken werden als Bedrohung für das Reich entworfen, die dieses aufgrund eigener Verfehlungen fürchten muss. Die Herrschaft der Türken funktioniert zwar besser als die des Reichs, aber sie ist, so macht das Spiel deutlich, indem es an der Vorstellung einer grundsätzlichen Überlegenheit des christlichen Glaubens festhält, ${ }^{83}$ einer funktionalen christlichen Regierung wie der in Nürnberg schon aus religiösen Gründen unterlegen.

Anders als dem türkischen Herrscher sind dem Kaiser im Spiel Des Entkrift Vafnacht (K 68) ${ }^{84}$ keine guten Ratgeber vergönnt. Der Text dramatisiert nach einer deutlich älteren alemannischen Vorlage ${ }^{85}$ in episodischer Reihung »wie

78 Die Finanzthematik ordnet Ehrstine: Fastnachtsrhetorik (s. Anm. 70), S. 16-17, anders ein.

79 Vgl. Isenmann: Die deutsche Stadt (s. Anm. 10), S. 325.

80 Linke: Aspekte der Wirklichkeits-Wahrnehmung (s. Anm. 9), S. 20.

81 Vgl. ebd., S. 25.

82 Vgl. Ragotzky: Der Bauer (s. Anm. 22), S. 97.

83 Vgl. Ackermann: Dimensionen der Medialität (s. Anm. 70), S. 206-207.

84 Im Folgenden zitiert nach: Frühe Schweizerspiele. Hg. von Friederike Christ-Kutter. Bern 1963, S. 30-61.

85 Vgl. Simon: Die Anfänge (s. Anm. 7), S. 36-38; Eckehard Simon: Geistliche Fastnachtspiele. Zum Grenzbereich zwischen geistlichem und weltlichem Spiel. In: Ingrid Kasten, Erika Fischer-Lichte (Hg.): Transformationen des Religiösen. Performativität und Textualität im geistlichen Spiel. Berlin u. a. 2007, S. 18-45, hier S. 20. 
der Antichrist nacheinander die Propheten Enoch und Elias, die Juden, den Kaiser, einen Bischof, Kaplan, schließlich einen widerstandleistenden Pilger töten lässt und/oder für sich gewinnt «. ${ }^{86}$ Im Hinblick auf den Zusammenhang von Rat und Regierung ist vor allem die Kaiser-Episode von Interesse: Der Antichrist wendet sich an den Kaiser und verspricht diesem Macht und Reichtum. Der davon verlockte Herrscher fragt daraufhin seine Ritter um Rat. Diese antworten nun ausführlich nach dem Prinzip der Rat-Erfragung: ${ }^{87}$

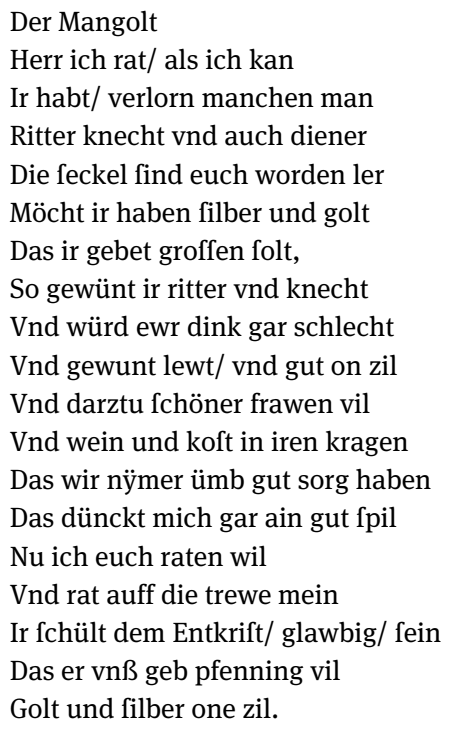

Zunächst raten drei Ritter dem Kaiser zu, das Angebot anzunehmen. Nur der letzte und vierte der befragten Ratgeber warnt seinen Herrn davor, seinen Glauben zu kompromittieren:

Der Degenhart

Da rat ich auff dÿ trewe mein Ir fchüllent vefft vnd ftēt fein An vnferm herrn Jhufu Crift Der von einer magt geporn ift Vnd habent Criftenlichen gelawben Des fchült ir nit verlawgen Wann ich offt/ gehört han

86 Glier: Rosenplütsche Fastnachtspiele (s. Anm. 9), Sp. 223.

87 Vgl. Linke: Aspekte der Wirklichkeits-Wahrnehmung (s. Anm. 9), S. 43. 


\author{
Das es nit wol ift gethan \\ Wer glawbet an den Entkrift \\ Wann er ain falfcher man ift \\ Der Kaÿfer \\ Dem merern taÿl ich volgen fchol \\ Wann es tzÿmpt dem weifen wol.
}

(V. 282-295)

Der Kaiser folgt der Mehrheitsmeinung und nicht dem richtigen und weisen Rat Degenharts. ${ }^{88}$ Damit erweist er sich als schlecht beratener und somit auch als schlechter Herrscher. Ausschlaggebend ist dafür zum einen die Unfähigkeit des Kaisers, auf den richtigen Rat zu hören, und zum anderen die Tatsache, dass ihm mehrheitlich schlechte Ratschläge erteilt werden. Der falsche Rat und der falsche Umgang mit den Ratschlägen werden zum Ausweis für die politischen Unzulänglichkeiten des Reichs, die hier religiös rückgebunden erscheinen und an die Dimension des Heils herangeführt werden. Der Antichrist wird, anders als für diesen geistlichen Spieltypen üblich, am Ende nicht gestürzt. ${ }^{89}$ Vor dem Hintergrund des bedrohlich-apokalyptischen Szenarios wird im Hinblick auf die Figur des Kaisers illustriert, dass schlechter Rat Unheil bedeutet. Dabei spielt die Verkehrung des Glaubens ${ }^{90}$ in doppelter Hinsicht eine entscheidende Rolle: Der Kaiser schenkt den falschen Ratgebern Glauben und er verleugnet seinen christlichen Glauben, indem er dem Antichrist folgt. So wird die politische Dimension im Sinne der Eschatologie erweitert. Damit wird ex negativo deutlich gemacht, dass die Bevölkerung der Reichsstadt im Hinblick auf das politische und religiöse Heil gut beraten ist, wenn sie an ihre Regierung glaubt bzw. ihrem Rat Glauben schenkt.

88 Vgl. Hedda Ragotzky: Fastnacht und Endzeit. Zur Funktion der Antichrist-Figur im Nürnberger Fastnachtspiel des 15. Jahrhunderts. In: ZfdPh 121 (2002), S. 54-71, hier S. 59-60.

89 Vgl. Klaus Ridder und Ulrich Barton: Die Antichrist-Figur im mittelalterlichen Schauspiel. In: Wolfram Brandes, Felicitas Schmieder (Hg.): Antichrist. Konstruktionen von Feindbildern. Berlin 2010, S. 179-195, hier S. 187-190.

90 Zum Antichrist als Figur der vollkommenen Verkehrung vgl. Robert W. Scribner: Reformation, Karneval und die sverkehrte Weltı. In: Richard van Dülmen, Norbert Schindler (Hg.): Volkskultur. Zur Wiederentdeckung des vergessenen Alltags (16.-20. Jahrhundert). Frankfurt a.M. 1984, S. 117-152, hier S. 150. 


\section{Fazit}

Literarische Darstellungen der Nürnberger Regierung sind im Spätmittelalter nicht ohne die reichsstädtische Zensurpolitik zu erklären, deren Geschichte sich

von der Mitte des 15. Jahrhunderts an als eine ständig weiter ausgreifende und immer perfekter werdende Kontrolle, als zunehmende Verschärfung der Auflagen und Restriktionen und als sich permanent vergrößernde Empfindlichkeit und Angst des Rats in allen von der Literatur berührten politischen und sozialen Fragen ${ }^{91}$

charakterisieren lässt. Für Rosenplüts Schaffenszeitraum war diese zwar noch deutlich weniger restriktiv als zur Zeit von Hans Folz und Hans Sachs. ${ }^{92}$ Dennoch scheint wie für seine >Nachfolger auch für Rosenplüt eine positive Darstellung des Rats kaum verwunderlich. Allerdings ist damit über deren besondere Form noch nicht viel gesagt. Diese im Sinne einer funktionsorientierten Analyse des Text-Kontext-Verhältnisses genauer zu erfassen, war deshalb Ziel dieses Beitrags. Verdeutlicht werden sollte, dass Rosenplüts literarische Darstellungen des Rats an gouvernementale Vorstellungen angelehnt sind, bei denen der Rat nicht als Souverän, sondern als Vertrauter erscheint, der nicht auf die Disziplinierung der Bevölkerung, sondern auf deren Beratung setzt. Indem die Texte dem Rat einen gouvernementalen Regierungsstil zusprechen, wirken sie auf die Steigerung seiner Popularität hin und lassen sich im Hinblick auf ihren Gehalt als ideologisch und im Hinblick auf ihre funktionale Ausrichtung als propagandistisch verstehen. Im Lobspruch auf Nürnberg wird diese gouvernementale Propaganda Rosenplüts durch den Entwurf des Stadtrats als Hirte in programmatischer Form greifbar. Der Rat erscheint dabei nicht als autokratische Institution, die auf Durchsetzung von Gesetz und Ordnung zielt, sondern als eine um Schutz und Zusammenhalt der Herde bemühte pastorale Instanz. In seinen Spielen nutzt Rosenplüt den dramatischen Modus, um den Rat über die Dimension der Kommunikation als unterstützend-solidarisch handelnde Instanz darzustellen. Der Nürnberger Rat erscheint dabei nicht als Behörde, Institution oder statische Systemstelle der politischen Ordnung oder des Rechtssystems, sondern wird als Ansprechpartner dargestellt, der um Ausgleich zwischen verschiedenen Interessensgruppen in der Stadt ebenso bemüht ist wie um unbürokratische Lösungsorientiertheit im Hinblick auf Anliegen der Bevölkerung. Die Kompetenz des Rats wird nicht durch die

91 Reichel: Der Spruchdichter (s. Anm. 5), S. 161.

92 Vgl. ebd., S. 161-165. 
Inszenierung eines autoritativen Regierungsstils illustriert, sondern über seine Nähe zu den Nürnbergerinnen und Nürnbergern, für die er als Ratgeber fungiert. Mittels der Darstellung fremder Regierungen verweist Rosenplüt zudem auf die Qualität der eigenen Regierung, wobei religiöse Dimensionen relevant werden. Die Idealität des Nürnberger Rats wird damit letztlich auch über eine Logik des Heils propagiert. 
